\title{
Probing electron dynamics by IR+XUV pulses
}

\author{
P.-G. Reinhard ${ }^{1}$ and Éric Suraud ${ }^{2,3,4, a}$ \\ 1 Institut für Theoretische Physik, Universität Erlangen, Staudtstraße 7, D-91058 Erlangen, Germany \\ ${ }^{2}$ Université de Toulouse, UPS, Laboratoire de Physique Théorique, IRSAMC, F-31062 Toulouse Cedex, France \\ 3 CNRS, UMR5152, F-31062 Toulouse Cedex, France \\ 4 School of Mathematics and Physics, Queen's University, Belfast, UK
}

Received 24 January 2020 / Received in final form 8 April 2020

Published online 4 August 2020

(C) The Author(s) 2020. This article is published with open access at Springerlink.com

\begin{abstract}
By recording observables of electron emission we analyze the response of small metal clusters and organic molecules to a pump probe setup using an IR fs laser pulse as pump followed by an attosecond XUV pulse as probe. As tool for the study, we use Time Dependent Density Functional Theory (TDDFT) in real time complemented by a simple 2-level model for principle effects. As observables, we consider total ionization, average kinetic energy from Photo Electron Spectra (PES) and anisotropy parameters from Photo-electron Angular Distributions (PAD). We show that these signals can provide a map of the system's dynamical properties. The connection is especially simple for metal clusters in which the response is dominated by the Mie surface plasmon. The case of organic molecules is more involved due to the considerable spectral fragmentation of the underlying dipole response. But at least the dipole anisotropy from PAD provides a clean and robust signal which can be directly associated to system's properties even reproducing non-linear effects such as the change of spectra with excitation strength.
\end{abstract}

\section{Introduction}

Thanks to major developments in laser technology, the analysis of photo induced ultrafast electron dynamics in clusters and molecules has made great progress over the last decades [1-3]. The availability of attosecond pulses $\left(1 \mathrm{as}=10^{-18} \mathrm{~s}\right)$, attained in high harmonic generation processes, promises to allow fully time-resolved analysis of electron dynamics. This brings an invaluable perspective complementing standard spectroscopic analysis [4]. Understanding ultrafast electronic processes such as timeresolved valence electronic motion [5], or bond breaking, is essential, for example to control chemical reactivity, but also in astrochemistry or even solar cell developments [6]. Attosecond pulses have also been used to address other fundamental phenomena such as electron tunneling [7] or photo-emission delay effect [8]. Beyond such fundamental questions, there are also numerous applications of ultrafast irradiation processes in many mechanisms such as vision or photosynthesis. They are also crucial to understand radiation damage of biomolecules [9].

Standard two-color attosecond experimental setups mix an infrared (IR) with an extreme ultraviolet (XUV) pulse. Such pump probe combinations often use a non-

\footnotetext{
* Contribution to the Topical Issue "Atomic Cluster Collisions (2019)", edited by Alexey Verkhovtsev, Pablo de Vera, Nigel J. Mason, Andrey V. Solov'yov.

${ }^{a}$ e-mail: suraud@irsamc.ups-tlse.fr
}

ionizing IR laser pulse as pump, which induces electronic motion probed (with controllable delay) by an ionizing attosecond single XUV pulse or pulse train [5,10-12]. Conversely, short XUV pulses can be used as pump (with typical duration in the 100 as range) with a phase controlled IR pulse as probe. The latter setups include streaking $[13,14]$ and RABBIT $[15,16]$ techniques. The majority of experiments uses signals from electron emission as a function of delay time between pump and probe, hopefully giving access to the system's properties. Ionization can be measured as such or energy resolved as in Photo-Electron Spectra (PES) [17-20] or even in an angular/energy resolved manner [11]. The (energy integrated) Photo-emission Angular Distribution (PAD), is also a fruitful observable $[21,22]$, which is known to deliver interesting information already for femtosecond pulses [23-25].

Numerous of such two-color experiments have focused on atoms $[11,26-28]$ and small molecules [29-33]. A number of experiments use a long IR pump (several tens of fs) and an XUV attosecond probe. The latter can be a single pulse, but is often represented by a train of XUV pulses synchronized with the IR frequency. Whatever the target system (atoms, small molecules) and whatever the observables (net ionization, PES, or combination PES/PAD) the striking result delivered by these measurements is a regular modulation of the ionization signal by half an IR period $[11,26,32]$. The analysis of this behavior has been attacked at various levels of sophistication, from schematic, (semi) analytical methods [34] to realistic 
ones [35] and all these models usually reproduce the observed oscillatory pattern pretty well. The seemingly generic feature of these oscillations nevertheless raises the question of how much specific properties of a given system can be practically extracted [36]. We recently revisited this question combining a simple analytical model with realistic Time-Dependent Density Functional Theory (TDDFT) [37-39] calculations of atoms, clusters and molecules in order to disentangle generic properties of the laser-electron dynamics from system's specific properties [40]. This analysis allowed to understand in a simple manner the basic oscillatory pattern observed experimentally. To a large extent, they are dominated by the interaction between the IR+XUV pulse and the free electron cloud, even in the most favourable setups in which one considers only a single XUV pulse rather than a train. System's specific properties are, nonetheless, present in the considered signals (net ionization, PES and PAD) but not easily accessible and thus require heavily model dependent analysis [40]. This makes the above pump probe setups not ideally suited to directly access system's properties in a robust manner.

In the present paper we thus investigate an alternative scenario with the aim to access system's properties in the most robust and simple way. This alternative pump and probe setup was considered in [4], in which the dominance of the IR laser frequency is reduced by considering a few cycle IR pulse, which delivers the excitation but does not imprint the IR frequency too strongly into the system's response thanks to the shortness of the pulse. Correlatively, using only one ultrashort XUV probe again avoids frequency bias as was imprinted in the earlier studies by the atto train. It was shown that such a setup provides interesting insights into details of ionization dynamics although most studies were performed with schematic models [4]. Such a setup thus appears as an ideal test case to explore how much one can access system's specific properties once the actual response is treated in a fully realistic manner. In the following we shall thus focus on such setups using a very short IR pulse (of order $1 \mathrm{fs}$ ) and one single short XUV probe (of order some 100 as). We shall consider for our study two different kinds of systems: $\mathrm{Na}_{9}^{+}$as a strongly polarizable metal cluster and $\mathrm{C}_{2} \mathrm{H}_{2}$ for a simple organic molecule. We use Time-Dependent Density Functional Theory (TDDFT) [37-39] to describe the electronic dynamics of the cluster or molecule $[19,22,38]$. For an introductory exploration, we shall also briefly refer to a simple schematic 2-level model recently introduced to analyze IR pump/XUV probe setups [40]. The paper is organized as follows. Section 2 provides a quick description of theoretical tools. The results are presented and discussed in Section 3.

\section{Theoretical framework}

\subsection{Laser setup}

We focus here on scenarios in which we pump the target system with a short IR pulse and probe it with an even shorter XUV pulse. As both pulses have wavelengths

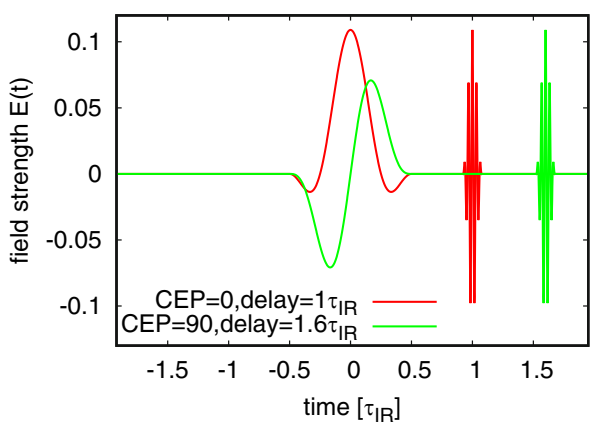

Fig. 1. Illustration of typical $I R+X U V$ pulses. Two cases with different carrier-envelope phase (CEP) and delay time are shown. The infrared frequency is $\omega_{\mathrm{IR}}=0.1 \mathrm{Ry}$ with cycle time $\tau_{\mathrm{IR}}=3.04 \mathrm{fs}$. The XUV frequency is $\omega_{\mathrm{X}}=3 \mathrm{Ry}$ and pulse length $T_{\mathrm{X}}=\tau_{\mathrm{IR}} / 6=0.51 \mathrm{fs}$. The intensities are $I_{\mathrm{IR}}=I_{\mathrm{X}}=10^{14} \mathrm{~W} / \mathrm{cm}^{2}$ corresponding to fields strengths $E_{\mathrm{IR}}=E_{\mathrm{X}}=0.109 \mathrm{Ry} / \mathrm{a}_{0}$.

much larger than the system's sizes, we work in the dipole approximation. The external photon field can then be written as

$$
U_{\text {las }}(\mathbf{r}, t)=-\mathbf{r} \cdot\left(\mathbf{F}_{\mathrm{IR}}(t)+\mathbf{F}_{\mathrm{X}}(t)\right)
$$

with

$$
\begin{aligned}
& \mathbf{F}_{\mathrm{IR}}(t)=\mathbf{E}_{\mathrm{IR}} f_{\mathrm{IR}}\left(\frac{t}{T_{\mathrm{IR}}}\right) \cos \left(\omega_{\mathrm{IR}} t\right), \\
& \mathbf{F}_{\mathrm{X}}(t)=\mathbf{E}_{\mathrm{X}} f_{\mathrm{X}}\left(\frac{t-t_{\mathrm{d}}}{T_{\mathrm{X}}}\right) \cos \left(\omega_{\mathrm{X}}\left(t-t_{\mathrm{d}}\right)\right),
\end{aligned}
$$

where $\tau_{\mathrm{IR}}=2 \pi / \omega_{\mathrm{IR}}$ is the IR period and $f$ is a cosine-squared envelope. The quantities $E_{\mathrm{IR}}, E_{\mathrm{X}} T_{\mathrm{IR}}, T_{\mathrm{X}}$, $\omega_{\mathrm{IR}}, \omega_{\mathrm{X}}$, are peak electric fields, pulse duration, and frequency of the IR and XUV pulses respectively. Finally the key quantity $t_{\mathrm{d}}$ is the delay between IR and XUV pulses, measured from center of peak of IR pulse to center of peak of XUV pulse. Both IR and XUV pulses are assumed linearly polarized along the $z$-direction. In the following, we will quantify the fields strengths $E$ in terms of pulse intensity $I \propto E^{2}$ because this is commonly used.

Figure 1 illustrates the IR+XUV pulses used for exciting the systems. The standard pulse parameters used throughout this paper are: IR frequency $\omega_{\mathrm{IR}}=0.1 \mathrm{Ry}$ corresponding to cycle time $\tau_{\mathrm{IR}}=3.04 \mathrm{fs}$; IR pulse length $T_{\mathrm{IR}}=\tau_{\mathrm{IR}} ; \mathrm{XUV}$ frequency $\omega_{\mathrm{X}}=3$ Ry; XUV pulse length $T_{\mathrm{X}}=\tau_{\mathrm{IR}} / 6=0.51 \mathrm{fs}$ which means $5 \mathrm{XUV}$ cycles in the pulse. Because of the short IR pulse length $T_{\mathrm{IR}}=\tau_{\mathrm{IR}}$ the Carrier Envelop Phase (CEP), namely the phase of the oscillatory pattern with respect to pulse envelop, acquires a large influence [23-25]. We shall briefly discuss its impact in Section 2.3.

\subsection{Electron dynamics by time-dependent density-functional theory and in a schematic model}

\subsubsection{Realistic TDDFT simulations}

Our basic tool to describe electron dynamics is TimeDependent Density-Functional Theory (TDDFT) [37-39]. 
We use it at the level of the Time-Dependent LocalDensity Approximation (TDLDA) with the exchange correlation functional of Perdew and Wang [41]. As pure LDA does not produce a correct Ionization Potential (IP) (violation of Koopmann's theorem), we augment it by a self-interaction correction (SIC) $[42,43]$ in a simplified density-averaged version (ADSIC) [44]. The latter turns out to be both reliable and efficient leading to a proper description of the ionization threshold for a great variety of systems [45].

The state of the system is represented in terms of singleparticle (s.p.) wave functions $\left\{\varphi_{i}(\mathbf{r}, t), i=1, \ldots, N\right\}$ where $N$ is the number of active electrons (i.e. valence electrons). The dynamics of single-electron wave functions is governed by the time-dependent Kohn-Sham equations (with atomic units $\hbar=1, e=1, m_{\mathrm{e}}=0.5$ )

$$
\begin{aligned}
i \partial_{t} \varphi_{i}(\mathbf{r}, t) & =\left(-\Delta+U_{\mathrm{KS}}(\mathbf{r}, t)+U_{\text {las }}(\mathbf{r}, t)\right) \varphi_{i}(\mathbf{r}, t) \\
U_{\mathrm{KS}}(\mathbf{r}, t) & =U_{\mathrm{ion}}(\mathbf{r})+\frac{\delta E[\varrho]}{\delta \varrho} \\
\varrho(\mathbf{r}, t) & =\sum_{i=1}^{N}\left|\varphi_{i}(\mathbf{r}, t)\right|^{2}
\end{aligned}
$$

where the Kohn-Sham potential $U_{\mathrm{KS}}$ is composed of the potential of the ionic background $U_{\text {ion }}$ and the electronic contribution from Coulomb (Hartree) and exchangecorrelation terms. It can be deduced as functional derivative of the energy-density functional $E[\varrho]$ with respect to the one-electron density $\varrho$. The ionic background $U_{\text {ion }}$ is provided by pseudopotentials, Goedecker-type [46] for Carbon and Hydrogen and a soft local pseudopotential for sodium [47]. These pseudopotentials have been checked to reproduce the relevant basic properties (ionization potential (IP), single-electron energies) sufficiently well in connection with ADSIC. Because of the very short time scales involved we keep the ionic background frozen at the initial equilibrium geometry.

The TDLDA equations are solved numerically on a grid in coordinate space with grid spacing $0.4 \mathrm{a}_{0}$ for the $\mathrm{Na}_{9}^{+}$as well as for the $\mathrm{C}_{2} \mathrm{H}_{2}$ molecule. The box length from origin to box bounds are $80 \mathrm{a}_{0}$ for $\mathrm{Na}_{9}^{+}$and $40 \mathrm{a}_{0}$ for $\mathrm{C}_{2} \mathrm{H}_{2}$, adapted to be much larger than the extension of the least bound electron. The static iterations towards the electronic ground state are done with the damped gradient method [48] and the time evolution employs the time-splitting technique [49] with a step size of $0.1 / \mathrm{Ry}=0.484$ as for $\mathrm{Na}_{9}^{+}$and $0.02 / \mathrm{Ry}=0.0968$ as for $\mathrm{C}_{2} \mathrm{H}_{2}$. To account for ionization, absorbing boundary conditions are implemented using a mask function [50]. The absorbing margin extends over $8 \mathrm{a}_{0}$ at each side of the grid. Results shown here were done on an axial grid which is still exact for the linear $\mathrm{C}_{2} \mathrm{H}_{2}$ molecule and a well tested approximation for $\mathrm{Na}_{9}^{+}$[51]. For more details on the numerical method see $[19,22,52]$.

\subsubsection{A complementing simple model viewpoint}

The TDDFT computations for realistic systems are complemented by results from a schematic model consisting of two bound levels coupled to a one-dimensional electron continuum [40]. The model relies on the Single Active Electron (SAE) model in the Strong Field (SF) approximation. The dynamics of the (free) continuum electrons is then assumed to be dominated by the laser field and the Coulomb potential can be neglected. The unperturbed system consists of $L$ bound states $|j\rangle$ and plane waves for the electron continuum $|\mathbf{p}\rangle$; its Hamiltonian is denoted $\hat{H}_{0}$. The dynamical electron wavefunction is then represented as

$$
\begin{aligned}
& |\Psi(t)\rangle=\sum_{j=1}^{L} c_{j}(t) e^{-i \varepsilon_{j} t}|j\rangle+\int d^{3} \mathbf{p} b(\mathbf{p}, t)|\mathbf{p}\rangle, \\
& \hat{H}_{0}|j\rangle=\varepsilon_{j}|j\rangle, \quad \hat{H}_{0}|\mathbf{p}\rangle=\frac{\mathbf{p}^{2}}{2}|\mathbf{p}\rangle .
\end{aligned}
$$

The full Hamiltonian $\hat{H}=\hat{H}_{0}+U_{\text {las }}$ is obtained by adding the external laser field equation (1), the same as used for TDDFT. The time evolution is determined by the Schrödinger equation $i \partial_{t}|\Psi\rangle=\hat{H}|\Psi\rangle$. This leads to two coupled equations of motion for the amplitudes $b(\mathbf{p})$ and $c_{j}$

$$
\begin{aligned}
\dot{c}_{j}(t) & =i \mathbf{F}_{\mathrm{IR}} \cdot \sum_{l} \mathbf{d}_{j l} c_{l}(t), \\
\dot{b}(\mathbf{p}, t) & =\left[-\frac{i}{2} \mathbf{p}^{2}+\mathbf{F} \cdot \nabla_{\mathbf{p}}\right] b(\mathbf{p}, t)+i \sum_{j} \mathbf{F} \cdot \mathbf{d}_{\mathbf{p} j} c_{j}(t),
\end{aligned}
$$

where the dipole matrix element $\mathbf{d}_{j l}=\langle j|\mathbf{r}| l\rangle$ is assumed to be real and where $\mathbf{d}_{\mathbf{p} j}=\langle\mathbf{p}|\mathbf{r}| j\rangle$. In equation (6a), following experimental conditions, we exploit the fact that bound states are dominantly coupled by the IR laser field, thus neglecting their population change through XUV photoionization. We then take $c_{1}(-\infty)=1$ and $c_{2}(-\infty)=0$ as initial conditions and solve the equations of motion. Although an analytical solution is feasible in limiting cases, it is only enlightening for further simplified situations. We thus solve the full equations of motion numerically. Actually, we use the model with $L=2$ bound states where the energy difference $\Delta \varepsilon=\varepsilon_{2}-\varepsilon_{1}$ is the system property searched for. Furthermore, we restrict the dimension to 1 for the sake of simplicity, i.e. $\mathbf{p} \rightarrow p$, without loss of generality.

This schematic model provides a simple viewpoint on the considered systems and allows to simply identify generic features. For example setting $\mathbf{d}_{21}=0$ delivers a minimal model with one single bound state and no excited bound state. Except the ionization potential, it contains no information on the system and thus reduces to the dynamics of a free electron in the external field. Including $\mathbf{d}_{21}$ allows to evaluate the impact of the system's internal structure on the observed outcome, still from a rather generic viewpoint. We shall thus use this schematic model as a guideline to motivate our more specific TDDFT computations. In the version of the schematic model used here the energy unit is provided by the Ionization Potential (IP) which we identify with 1 Ry. The first bound state is the ground state and the second bound state has an excitation energy above ground state of $1 / 5$ of the IP. 


\subsection{Observables}

Following current experiments we shall focus on ionization properties. These can be analyzed at various levels of detail. The most global observable is the total ionization $N_{\text {esc }}$ emerging after applying the pulse. For our purpose the most important dependence is that on delay time $t_{\mathrm{d}}$ between IR and XUV pulses. We find as typical behavior of $N_{\text {esc }}\left(t_{\mathrm{d}}\right)$ oscillatory pattern overlaid with some global trend. The information content can most efficiently be visualized by taking the Fourier transform of $N_{\mathrm{esc}}\left(t_{\mathrm{d}}\right)$ and calculating the associated power spectrum $[32,40]$ :

$$
\mathcal{P}(\omega)=\left|\int d t_{\mathrm{d}} e^{i \omega t_{\mathrm{d}}}\left[N_{\mathrm{esc}}\left(t_{\mathrm{d}}\right)-N_{\mathrm{esc}, \operatorname{lin}}\left(t_{\mathrm{d}}\right)\right]\right|^{2}
$$

where $N_{\text {esc,lin }}\left(t_{\mathrm{d}}\right)$ subtracts a possible global linear trend, which could easily produce too large spectral background. The analysis in terms of $N_{\text {esc }}$ can be complemented by considering energetic properties of emitted electrons in terms of PES yield $\sigma_{\mathrm{PES}}\left(E_{\mathrm{kin}}\right)$ and angular properties in terms of angular distribution $\sigma_{\mathrm{PAD}}(\vartheta)[22,35]$. Again, PES and PAD will be studied as function of delay time $t_{\mathrm{d}}$. Thus it is simpler to quantify them in terms of a few numbers. We characterize the PES by the average kinetic energy

$$
\bar{E}_{\text {kin }}=\bar{E}_{\text {kin }}\left(t_{\mathrm{d}}\right)=\frac{\int d E_{\text {kin }} E_{\text {kin }} \sigma_{\mathrm{PES}}\left(E_{\mathrm{kin}}, t_{\mathrm{d}}\right)}{\int d E_{\mathrm{kin}} \sigma_{\mathrm{PES}}\left(E_{\mathrm{kin}}, t_{\mathrm{d}}\right)}
$$

and the PAD by its first two moments, namely the dipole $\beta_{1}$ and quadrupole $\beta_{2}$ anisotropy parameters [53,54].

$$
\beta_{i}=\frac{\int d \vartheta P_{i}(\cos (\vartheta)) \sigma_{\mathrm{PAD}}\left(\vartheta, t_{\mathrm{d}}\right)}{\int d \vartheta \sigma_{\mathrm{PAD}}\left(\vartheta, t_{\mathrm{d}}\right)}
$$

where $P_{i}$ are Legendre polynomials of order $i$. The dipole anisotropy $\beta_{1}$ is particulary sensitive to the left/right asymmetry of ionization flow (with respect to laser polarization) and the quadrupole anisotropy $\beta_{2}$ to the relation of longitudinal to sideward emission. All these numbers are functions of delay time $t_{\mathrm{d}}$ and we shall again analyze them in terms of the associated power spectra which are evaluated in the same manner as for the total ionization yield, see equation (7).

The evaluation of observables is considerably simpler for the schematic model. All observables are extracted from the asymptotic continuum amplitude $b(p, t=\infty)$. We consider in the following ionization, average momentum and average kinetic energy as (mind that we restrict ourselves to $1 \mathrm{D}$ for the computations performed within the schematic model)

$$
\begin{aligned}
N_{\mathrm{esc}} & =\int d p|p b(p, t=\infty)|^{2}, \\
\bar{p} & =\frac{\int d p p|b(p, t=\infty)|^{2}}{N_{\mathrm{esc}}}, \\
\bar{E}_{\mathrm{kin}} & =\frac{\int d^{3} p \mathrm{p}^{2}|b(p, t=\infty)|^{2}}{N_{\mathrm{esc}}},
\end{aligned}
$$

where $\bar{p}$ and $\bar{E}_{\text {kin }}$ serve as representatives for the PES.
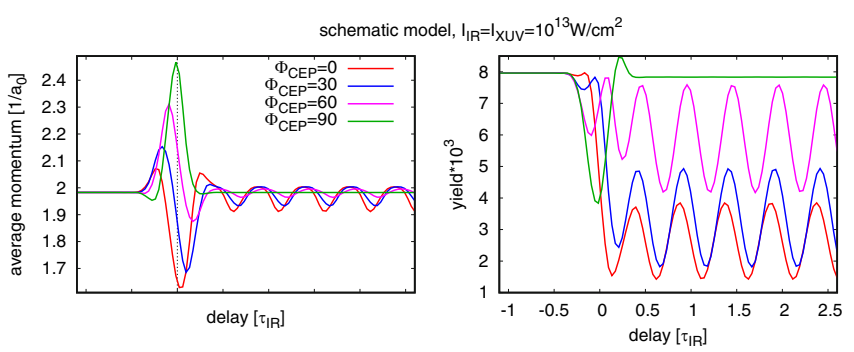

Fig. 2. Results from the schematic 2-state model [40] with electron continuum for total ionization (right) and average momentum $\bar{p}$ of emitted electrons (left) for various CEP as function of delay time.

As indicated above the CEP $\phi_{\mathrm{CEP}}$ of the IR pulse is a crucial parameter of the setup as the total duration of the IR pulse equals the IR period. Before proceeding we thus check the impact of the CEP. For the sake of simplicity, we do that using our schematic model [40] and consider as observable the average momentum of emitted electrons. The impact of the CEP is illustrated in Figure 2. Clearly the amplitude of the signal is largest, and thus easier to analyze, for $\phi_{\mathrm{CEP}}=0$. To understand that recall that the field strength represents the force on the electron cloud. The net force from a pulse with $\phi_{\mathrm{CEP}}=0$ is largest while it is practically zero for $\phi_{\mathrm{CEP}}=90^{\circ}$, as can be read off from Figure 1. In all forthcoming applications we have thus chosen $\phi_{\mathrm{CEP}}=0$ which was found to be most effective for the present purposes.

Figure 2 shows a further subtle detail. The phase of the asymptotic oscillations, after the pulse is over, depends slightly on the CEP, which reflects details of the systems immediate response to the IR pulse. As these are very subtle effects, we ignore them in the present first exploration.

\section{Results and discussion}

\subsection{Schematic model}

We start with a result from the schematic model because it delivers simple and clean signals while reproducing already typical effects [40]. To put the results into perspective, recall that the IP has been chosen at 1 Ry and excited bound state has 0.2 Ry excitation energy. Figure 3 shows total ionization and average kinetic energy for two different IR intensities, recorded as function of delay time $t_{\mathrm{d}}$ and transformed into the frequency domain. The figure displays several interesting pattern. First, one clearly sees that all signals display regular oscillations, whose frequency is the frequency corresponding to the energy difference between the two bound levels, which is twice the IR frequency. For one of the observables, namely the average kinetic energy, frequency doubling occurs and the amplitude of the effect clearly depends on IR intensity (recall that a 2-level system corresponds to motion in a cosine potential which causes anharmonicities with increasing amplitude). This shows the importance of both the nature of the observables and of the IR intensity. We shall thus 

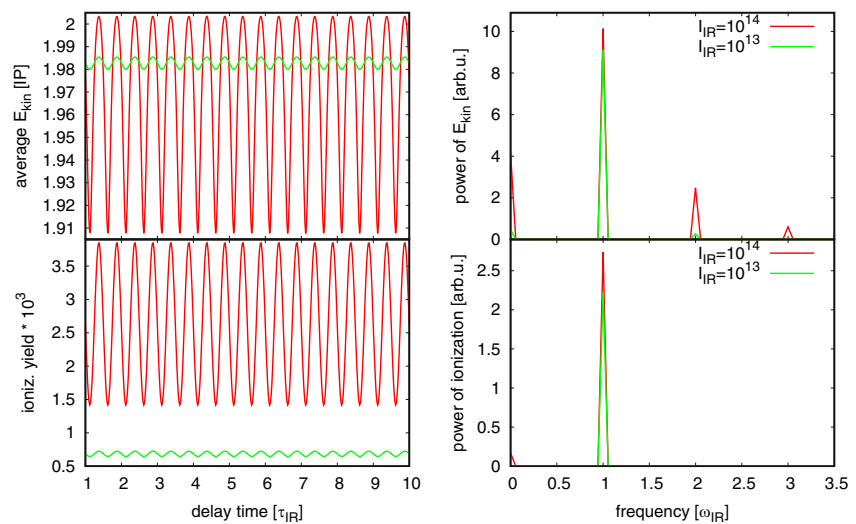

Fig. 3. Results from the schematic 2-state model [40] with electron continuum for two IR field intensities as indicated (in units $\left.\mathrm{W} / \mathrm{cm}^{2}\right)$. Left: signals as function of delay time. Right: corresponding signals in frequency domain. Lower: analysis from total ionization yield. Upper: analysis from average kinetic energy of emitted electrons.

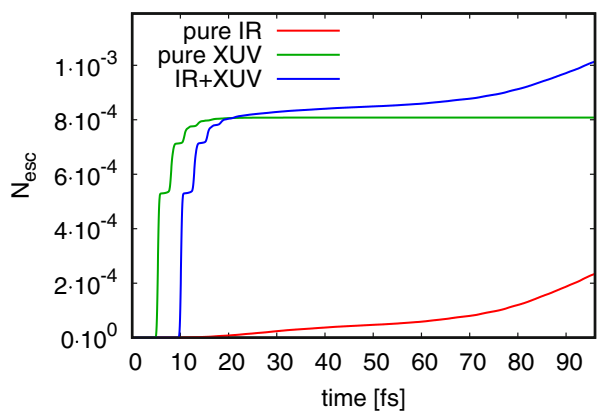

Fig. 4. Time evolution of ionization for $\mathrm{Na}_{9}^{+}$and three different excitations: pure IR pulse with field intensity $I_{\mathrm{IR}}=10^{11} \mathrm{~W} / \mathrm{cm}^{2}$ (corresponding to field strength $E_{\mathrm{IR}}=$ $0.0034 \mathrm{Ry} / \mathrm{a}_{0}$ ), pure XUV pulse with $I_{\mathrm{X}}=10^{15} \mathrm{~W} / \mathrm{cm}^{2}$ (corresponding to field strength $\left.E_{\mathrm{X}}=0.34 \mathrm{Ry} / \mathrm{a}_{0}\right)$, and a combination of both pulses. Otherwise, the standard pulse parameters of this paper are used (see text).

take care of considering both these aspects in the following. The schematic 2-level model has shown that the present IR+XUV setup can unravel properties of systems, at least with simple spectra. We shall thus pursue our analysis by considering such a highly polarizable system but now within the detailed realistic TDDFT approach.

\section{$3.2 \mathrm{Na}_{9}^{+}$as example for a metal cluster}

The basic idea of the IR+XUV setups is to analyze emission properties as a function of the delay time between IR and XUV pulses. In order to properly access such quantities we need to disentangle the ionization produced by the full IR+XUV combination from ionization generated separately by IR or XUV pulses. This is illustrated in Figure 4 focusing on the total ionization probability (for the relation of ionization probability to ionization see [52]). The weakly bound metal cluster allows some ionization already from the IR pulse. Although it is smaller than the yield
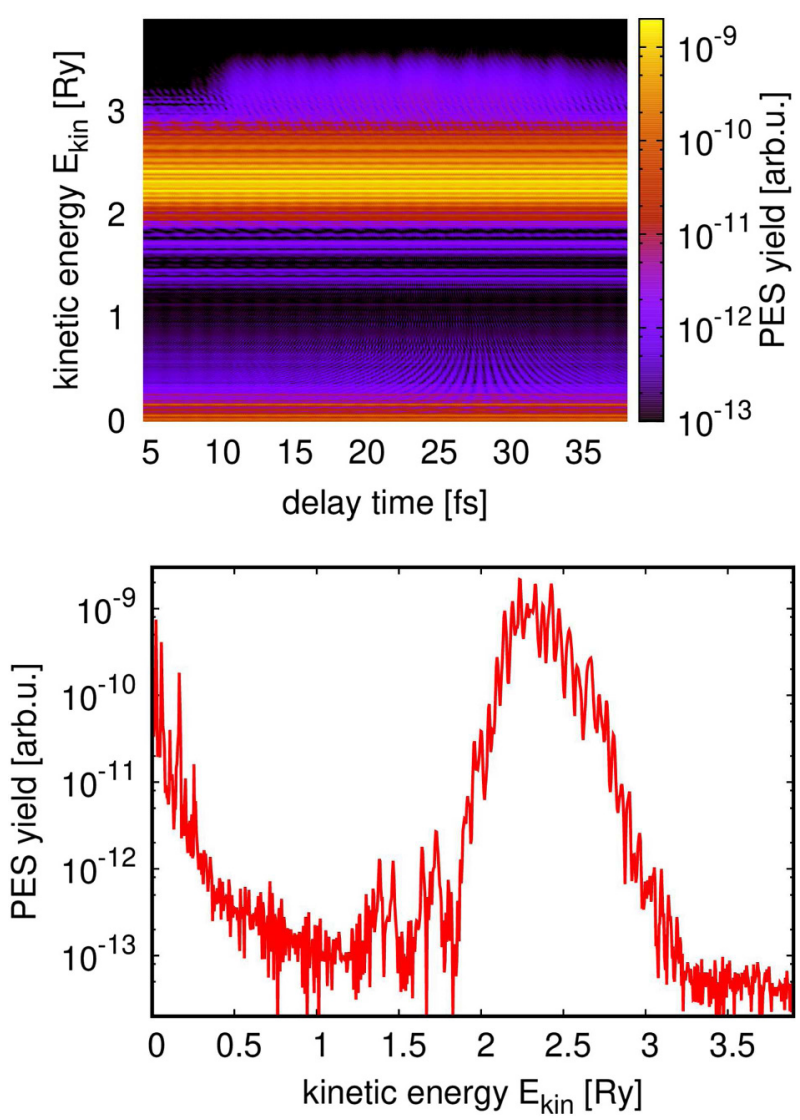

Fig. 5. Photo-electron spectra (PES) from IR+XUV excitation of $\mathrm{Na}_{9}^{+}$with standard pulse parameters and field strengths $I_{\mathrm{IR}}=10^{11} \mathrm{~W} / \mathrm{cm}^{2}$ and $I_{\mathrm{X}}=10^{15} \mathrm{~W} / \mathrm{cm}^{2}$. Lower: PES yield versus kinetic energy of emitted electron for delay time $4.84 \mathrm{fs}$. Upper: color map plot of the PES as a function of delay time.

from the XUV pulse, it is not negligible and needs consideration. We see that the separate IR plus XUV ionization yields together comes close to the yield from IR+XUV signal. We are thus after the faint differences between these two signals as function of delay time. This requires, in principle, to subtract the combined ionization of the pure pulses from the total ionization from the IR+XUV pulse. We will do that in the following for the signals as a function of delay time. However, the background contribution is constant in delay time and drops automatically in the power spectrum (excluding the point at $\omega=0$ ).

As argued above, PES promise to provide a richer signal than mere total ionization. We check the case in Figure 5. The lower panel displays one PES as such for a given value of the delay time. One immediately identifies a wide bump in the spectrum associated to the XUV frequency shifted by the IP [55]. The signal, dominated by XUV ionization (see Fig. 4) is smeared around its expectation value $\left(\mathrm{IP}+\omega_{X}\right)$ because of the extremely short duration of the XUV pulse. The upper panel displays a color-map plot of the PES as function of the delay time. All PES (vertical cut) look very similar to the one in the lower panel. At the given plotting scale it is impossible to spot a structure 

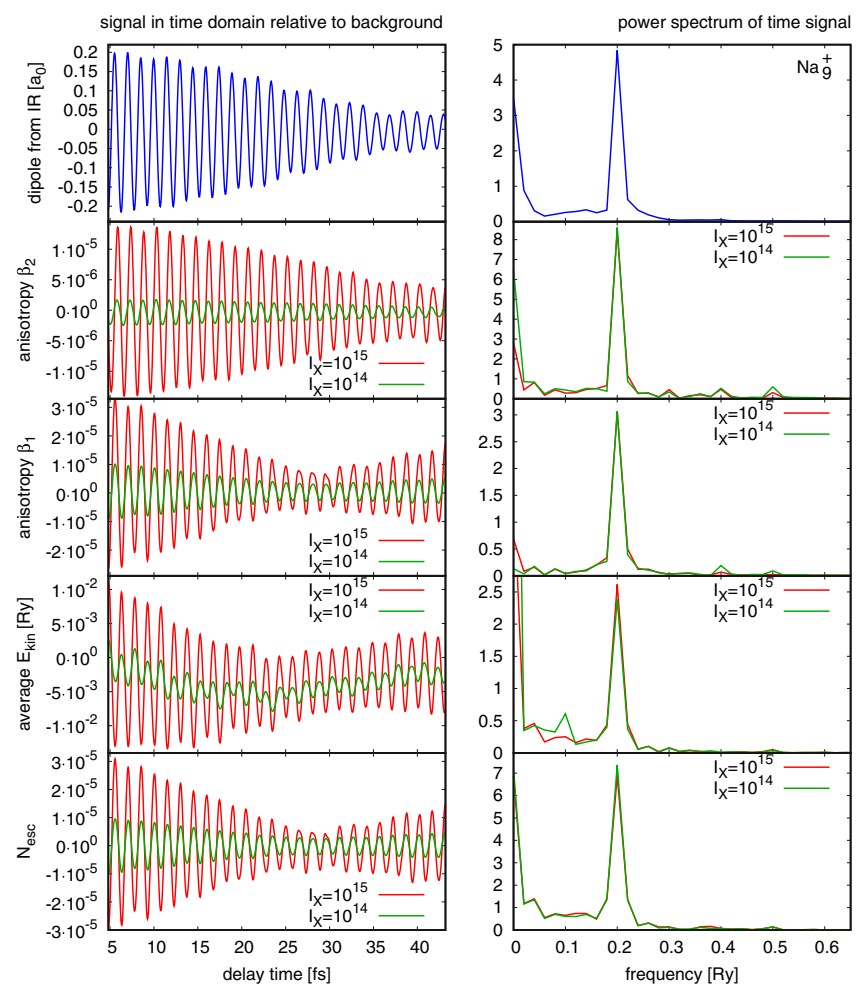

Fig. 6. Signals from different observables in time domain (left) and frequency domain (right) for $\mathrm{Na}_{9}^{+}$excited by IR+XUV pulses with standard pulse parameters, IR field intensity $I_{\mathrm{IR}}=$ $10^{11} \mathrm{~W} / \mathrm{cm}^{2}$, and two different XUV intensities as indicated (in units of $\mathrm{W} / \mathrm{cm}^{2}$ ). The observables are ionization yield $N_{\text {esc }}$, average kinetic energy $\bar{E}_{\text {kin }}$, and dipole anisotropy $\beta_{1}$ as well as quadrupole anisotropy $\beta_{2}$. These signals are drawn in the left panels as function of delay time. The right panels show the corresponding power spectra. The uppermost panels show dipole signal following pure IR excitation, left panel as function of time and right panel its power spectrum.

as a function of delay time. This clearly calls for cleaner/simpler indicators. The first natural step is to consider average quantities as simple numbers characterizing the ionization. We shall thus extract the average kinetic energy as function of delay time. A similar problem occurs for the PAD and we will use the asymmetry parameters $\beta_{1}$ and $\beta_{2}$ as simple, integrated measures of PAD.

Figure 6 gives a summary of the behavior of our major dynamical indicators (total ionization, average kinetic energy $\bar{E}_{\text {kin }}$, dipole anisotropy $\beta_{1}$ and quadrupole anisotropy $\beta_{2}$ from bottom to top). The left column displays the indicators as a function of delay time $t_{\mathrm{d}}$. The right column provides the corresponding power spectra. Finally, the 2 upper panels display the dipole response following a pure IR pulse, left the time evolution and right the associated power spectrum [56]. The latter shows clearly the surface plasmon peak which is particularly clean in this small system. In the time domain (left panels), each observable delivers regular oscillatory pattern on top of a general trend, especially in the case of the average kinetic energy. Power spectra (right panels) provide the frequency content. The figure delivers a very clear message with all signals dominated by a peak at the plasmon frequency. The zero energy component reflects the global trend of each observable and is irrelevant for the present purpose. Changing the IR intensity changes the amplitude of the signals in real time but does not alter the spectral analysis. We thus recover, in this realistic case, most of what we had observed in the schematic 2-level model (Fig. 3). We shall come back later on the impact of IR and XUV intensities with respect to each other.

The case of $\mathrm{Na}_{9}^{+}$thus appears quite simple and clear. The IR+XUV setup immediately delivers signals basically reflecting a crucial system property, the dominating plasmon frequency. After all this may not be so surprising. Using a very short IR pulse provides a broad energy band so that the dominating plasmon frequency is immediately attached and the electron cloud oscillates accordingly. The XUV probe allows to analyze this oscillation in real time which is reflected in the associated spectral analysis. The perfect match between the response to the probe and the plasmon reflects the fact that the excitation spectrum of the system is dominated by one single frequency. This pump and probe setup thus clearly gives access to a system's specific property, even if one has to admit that the dominating property, the plasmon, is accessible in many other, less complex, ways as, e.g. photo-absorption measurements [57]. At second glance, we realize that there are new aspects in the result which cannot be accessed otherwise. For example, the signals in the time domain (left panels) demonstrate clearly the change in pattern with changing laser intensity, a clear signal of non-linear effects in the systems response. Thus far the situation with one dominating plasmon mode. It may become more involved in cases with a richer spectral structure. This is what we shall now explore using in the next example a small organic molecule.

\section{3 $\mathrm{C}_{2} \mathrm{H}_{2}$ as example for an organic molecule}

We now proceed to the case of a small organic molecule, namely $\mathrm{C}_{2} \mathrm{H}_{2}$, which has a similar number of valence electrons as $\mathrm{Na}_{9}^{+}$but a linear structure at variance with the spherical shape of $\mathrm{Na}_{9}^{+}$. We perform the same type of analysis as in the case of $\mathrm{Na}_{9}^{+}$and present the results in Figure 7 in exactly the same manner as in Figure 6. The figure shows that the IR+XUV analysis is not that straightforward for an organic molecule with its more fragmented dipole spectrum (see upper right panel). Different observables produce quite different power spectra and most of them are more involved than the simple dipole spectrum. This is true for all signals except for the emission asymmetry $\beta_{1}$.

In order to try to analyze the observed signals we have also considered higher multipole responses than the mere dipole. The quadrupole spectrum from pure IR pulse is thus also displayed in the right upper panel but it does not bring new insight. The striking feature concerns the appearance of a strong peak at twice the frequency of the strongest dipole peak in the spectrum. This indicates frequency doubling similar as we had seen in the schematic 

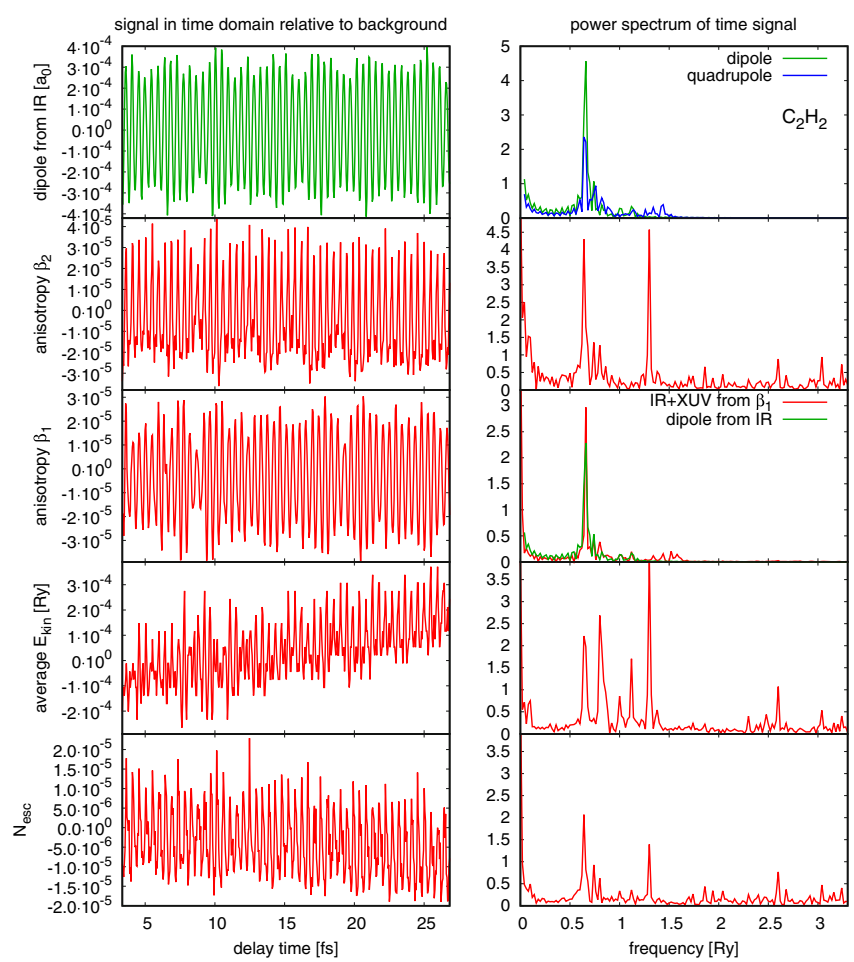

Fig. 7. Signals from different observables in time domain (left) and frequency domain (right) for $\mathrm{C}_{2} \mathrm{H}_{2}$ excited by IR+XUV pulses with standard pulse parameters and field strengths $I_{\mathrm{IR}}=10^{13} \mathrm{~W} / \mathrm{cm}^{2}$ with $I_{\mathrm{X}}=10^{14} \mathrm{~W} / \mathrm{cm}^{2}$. The observables are ionization yield $N_{\text {esc }}$, average kinetic energy $\bar{E}_{\text {kin }}$, and dipole anisotropy $\beta_{1}$ as well as quadrupole anisotropy $\beta_{2}$. These signals are drawn in the left panels as a function of delay time. The right panels show the corresponding power spectra. The uppermost panels show dipole signal following pure IR excitation, left panel as function of time and right panel its power spectrum. The upper right panel shows in addition the power spectrum of the quadrupole following pure IR excitation.

model, Figure 3. Now consider that the whole dipole spectrum (upper right panel), possibly also with contributions of the quadrupole spectrum, is frequency doubled by folding the pure IR spectra with themselves. This can, indeed, explain qualitatively the complexity of the spectra from a superposition of dipole and quadrupole contributions as modeling the yield as $\mathcal{Y}=c_{D}\left(D_{0}+D(t)\right)^{\gamma_{D}}+c_{Q}\left(Q_{0}+\right.$ $Q(t))^{\gamma_{Q}}$. This matches our former analysis on the structure of PES where we had seen that the complex PES spectra could be understood as a superposition of various multipoles in the case of complex IR+XUV setups [55]. This argument, though, is qualitative and calls for more detailed analysis of such involved spectra.

Still, one should not overlook the fact that, contrarily to other signals, the dipole anisotropy $\beta_{1}$ displays a very clean signal by itself, directly matching the dipole response. The filter on asymmetry has managed to suppress the contributions from two-photon emission which is obvious because two-photon processes are reflection symmetric along the polarization axis. The outcome is particularly encouraging as $\beta_{1}$ is a rather simple and robust quantity to measure. It is well known that it provides a
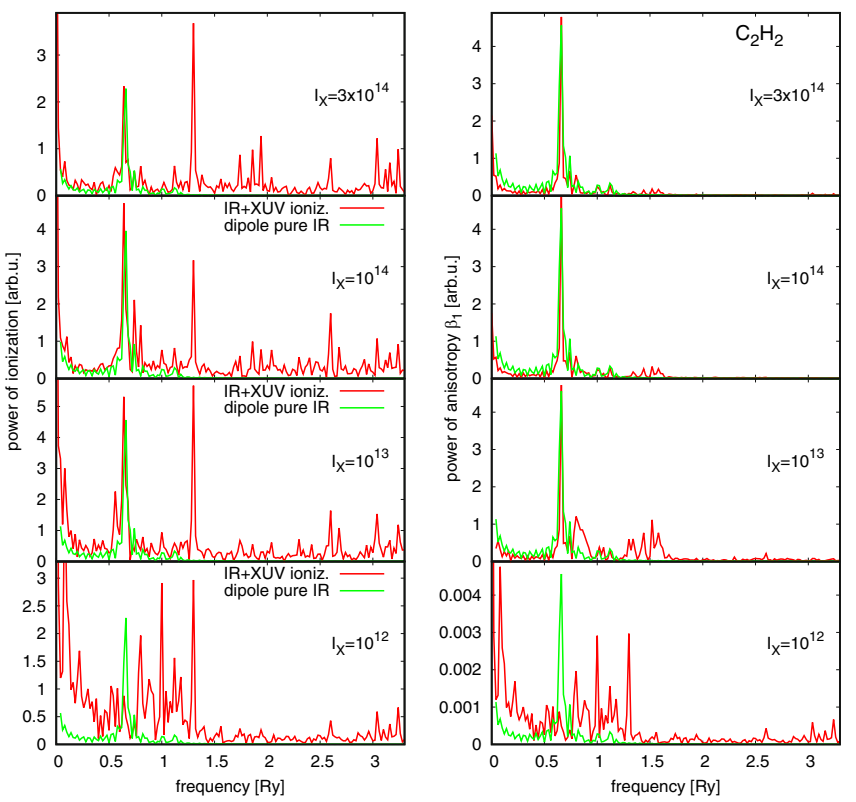

Fig. 8. Power spectra from IR+XUV analysis for $\mathrm{C}_{2} \mathrm{H}_{2}$ with the observables ionization (left panels) and dipole anisotropy $\beta_{1}$ (right panels) for IR $+\mathrm{XUV}$ excitation with standard pulse parameters and IR intensity $I_{\mathrm{IR}}=10^{13} \mathrm{~W} / \mathrm{cm}^{2}$. The XUV intensity is varied as indicated (in units of $\mathrm{W} / \mathrm{cm}^{2}$ ).

direct indicator of the CEP in the case of short IR pulses [58]. This promising result calls for further studies under varied conditions (systems, pulse parameters).

As a first step in that direction, we have performed a systematic investigation of the impact of the laser intensities. Results are displayed in Figure 8 where we consider various combinations of IR and XUV intensities, focusing on the emission asymmetry parameter $\beta_{1}$. The figure clearly demonstrates that a proper balance between IR field strength and XUV field strength is required to obtain clean signals. As a rule of thumb, the XUV strength should produce a response (in terms of emission) which is as large or larger than the yield from IR pulse. With such a setup the signal delivered by $\beta_{1}$ is especially clean and convincing. It thus clearly provides a simple and robust marker of the dynamical response of the system.

We round up this study with a variation of the IR intensity $I_{\mathrm{IR}}$. The guiding question emerging from our previous results is whether one can learn more on the dynamical response than the mere dipole spectra. More specifically, could $\beta_{1}$ offer an access to the non-linear response of the system? In order to explore this question we shall simply vary the intensity of the IR pulse which, in the non-linear domain where we are in the present example, changes the pattern of the dipole response and not only the amplitude. The XUV strength was chosen in both cases in the wanted regime of large emission (larger than emission from IR pulse). Figure 9 shows the change of power spectra with IR field strength. To be compared are the dipole spectra from a pure IR pulse (green) with the spectra deduced from IR+XUV analysis via $\beta_{1}$ (red). The dipole spectra show, as expected, significant dependence on IR strength reflecting the non-linear response [59]. The 


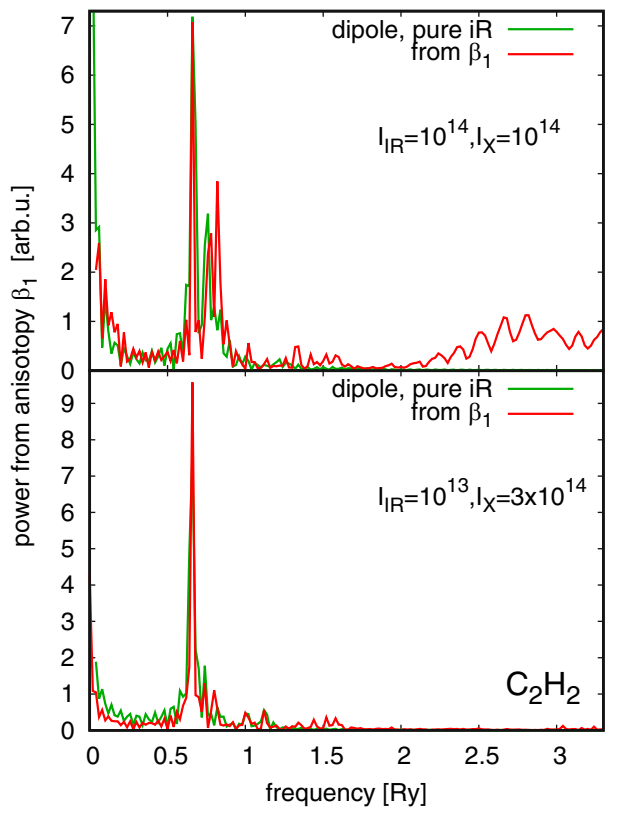

Fig. 9. Effect of variation of field strengths on power spectra from dipole anisotropy $\beta_{1}$ for IR+XUV excitation of $\mathrm{C}_{2} \mathrm{H}_{2}$.

gratifying result is that the changes in spectra are well reproduced by the power spectrum of the dipole anisotropy $\beta_{1}$. There still remain differences at high energies which are probably traces of multi-photon copies (probably three photons) of the dipole spectrum and which deserve further investigations. Still, these first results indicate in a convincing manner that the IR+XUV analysis can reproduce the non-linear effects of the system's response with changing field strength, and this on the basis of the simple and robust emission asymmetry parameter $\beta_{1}$. This last conclusion nicely complements the former one on the highly polarizable $\mathrm{Na}_{9}^{+}$in which the plasmon was overdominant. We seem, indeed, to gain access to a subtle quantity closely related to the non-linear dynamics of the system. The covalent case, however, is more involved and will require theory-supported analysis to unfold all dynamical features.

\section{Conclusions}

Using time-dependent density-functional theory, we have studied the analysis of the dynamical response of small electronic systems by an IR+XUV pump probe setup combining a short pump femtosecond IR pulse with an attosecond probe XUV pulse and recording subsequent electron emission as function of the delay between IR and XUV pulses. We have considered several observables from electron emission, total ionization, Photo Electron Spectra (PES) and Photo-electron Angular Distributions (PAD). The latter two have been reduced to a few characteristic numbers (average kinetic energy for PES and dipole as well as quadrupole anisotropy for PAD) to track better the trends with delay time. The four signals have been inspected directly as function of delay time and, transformed into frequency space, as corresponding power spectra. Test cases were $\mathrm{Na}_{9}^{+}$for metallic clusters and $\mathrm{C}_{2} \mathrm{H}_{2}$ for an organic molecule complemented by a simple schematic model for first explorations.

We have shown that particularly the power spectra provide valuable information on the intrinsic properties of the system. The case of metal clusters is especially simple as the response of the system is dominated by the Mie surface plasmon with one prevailing frequency appearing clearly in all power spectra from IR+XUV analysis. One could also find a small secondary peak at double plasmon frequency depending on fields strength and observable.

The situation is more involved from the onset in organic molecules because already the simple dipole spectrum is much fragmented. Correspondingly, the power spectra from IR+XUV analysis display an overly rich multi-peak structure. Still, one can qualitatively explain their structure from superposition of one-photon and multi-photon contributions. To recover the underlying dipole spectra would require in general a model-dependent unfolding of the spectral mix. A gratifying exception is, however, the signal from dipole anisotropy which displays in the most relevant energy range a formidable map of the system's dipole spectrum. The reason is that reflection asymmetric dipole observable suppresses successfully contributions from two-photon processes. Varying the pulse intensities, we could show that the IR+XUV analysis through dipole anisotropy is even able to reproduce correctly the nonlinear drift of the dipole spectrum with IR intensity. The latter case is a clear demonstration that one can possibly access system's specific non-linear dynamical properties, beyond structural and spectral properties attained in experimental setups.

The first results worked out in this paper open an interesting line of time resolved analysis of electronic dynamics. It motivates further investigations with systematically varied conditions concerning pulses and systems.

We thank the RRZE (Regionales Rechenzentrum Erlangen) for supplying the necessary computing power on their HPC systems.

\section{Author contribution statement}

All authors have contributed in a balanced way in this paper.

Publisher's Note The EPJ Publishers remain neutral with regard to jurisdictional claims in published maps and institutional affiliations.

Open Access This is an open access article distributed under the terms of the Creative Commons Attribution License (https://creativecommons.org/licenses/by/4.0/), which permits unrestricted use, distribution, and reproduction in any medium, provided the original work is properly cited.

\section{References}

1. T. Brabec, F. Krausz, Rev. Mod. Phys. 72, 545 (2000)

2. F. Krausz, M. Ivanov, Rev. Mod. Phys. 81, 163 (2009) 
3. L. Young, K. Ueda, M. Gühr, P.H. Bucksbaum, M. Simon, S. Mukamel, N. Rohringer, K.C. Prince, C. Masciovecchio, M. Meyer, A. Rudenko, J. Phys. B 51, 032003 (2018)

4. R. Pazourek, S. Nagele, J. Burgdörfer, Rev. Mod. Phys. 87, $765(2015)$

5. E. Goulielmakis, Z.H. Loh, A. Wirth, R. Santra, N. Rohringer, V.S. Yakovlev, S. Zherebtsov, T. Pfeifer, A.M. Azzeer, M.F. Kling, S.R. Leone, Nature 466, 739 (2010)

6. A. Föhlisch, P. Feulner, F. Hennies, A. Fink, D. Menzel, D. Sanchez-Porta, P.M. Echenique, W. Wurth, Nature 436, 373 (2005)

7. M. Uiberacker, T. Uphues, M. Schultze, A.J. Verhoef, V. Yakovlev, M.F. Kling, J. Rauschenberger, N.M. Kabachnik, H. Schröder, M. Lezius, K.L. Kompa, Nature 446, 627 (2007)

8. M. Schultze, M. Fieß, N. Karpowicz, J. Gagnon, M. Korbman, M. Hofstetter, S. Neppl, A.L. Cavalieri, Y. Komninos, T. Mercouris, C.A. Nicolaides, Science 328, 1658 (2010)

9. A.V. Solov'yov, Nanoscale Insights into Ion-Beam Cancer Therapy (Springer, 2016)

10. J. Itatani, F. Quéré, G.L. Yudin, M.Y. Ivanov, F. Krausz, P.B. Corkum, Phys. Rev. Lett. 88, 173903 (2002)

11. P. Johnsson, R. López-Martens, S. Kazamias, J. Mauritsson, C. Valentin, T. Remetter, K. Varjú, M.B. Gaarde, Y. Mairesse, H. Wabnitz, P. Salières, Phys. Rev. Lett. 95, 013001 (2005)

12. T. Remetter, P. Johnsson, J. Mauritsson, K. Varjú, Y. Ni, F. Lépine, E. Gustafsson, M. Kling, J. Khan, R. LópezMartens, K.J. Schafer, Nat. Phys. 2, 323 (2006)

13. M. Drescher, M. Hentschel, R. Kienberger, G. Tempea, C. Spielmann, G.A. Reider, P.B. Corkum, F. Krausz, Science 291, 1923 (2001)

14. G. Sansone, E. Benedetti, F. Calegari, C. Vozzi, L. Avaldi, R. Flammini, L. Poletto, P. Villoresi, C. Altucci, R. Velotta, S. Stagira, Science 314, 443 (2006)

15. V. Véniard, R. Taïeb, A. Maquet, Phys. Rev. A 54, 721 (1996)

16. K. Klünder, J.M. Dahlström, M. Gisselbrecht, T. Fordell, M. Swoboda, D. Guenot, P. Johnsson, J. Caillat, J. Mauritsson, A. Maquet, R. Taïeb, Phys. Rev. Lett. 106, 143002 (2011)

17. C. Bordas, F. Paulig, H. Heln, D.L. Huestis, Rev. Sci. Instrum. 67, 2257 (1996)

18. T. Seideman, Annu. Rev. Phys. Chem. 53, 41 (2002)

19. P.G. Reinhard, E. Suraud, Introduction to Cluster Dynamics (Wiley, 2003)

20. T. Fennel, K.H. Meiwes-Broer, J. Tiggesbäumker, P.G. Reinhard, P.M. Dinh, E. Suraud, Rev. Mod. Phys. 82, 1793 (2010)

21. P.K. Ghosh, Introduction to Photoelectron Spectroscopy (John Wiley and Sons, New York, 1983)

22. P. Wopperer, P.M. Dinh, P.G. Reinhard, E. Suraud, Phys. Rep. 562, 1 (2015)

23. G.G. Paulus, F. Lindner, H. Walther, A. Baltuska, E. Goulielmakis, M. Lezius, F. Krausz, Phys. Rev. Lett. 91, 253004 (2003)

24. C.Z. Gao, P.M. Dinh, P.G. Reinhard, E. Suraud, C. Meier, Phys. Rev. A 95, 033427 (2017)

25. P.G. Reinhard, E. Suraud, C. Meier, J. Phys. B 51, 024007 (2017)
26. P. Johnsson, J. Mauritsson, T. Remetter, A. L'Huillier, K.J. Schafer, Phys. Rev. Lett. 99, 233001 (2007)

27. P. Agostini, L.F. DiMauro, Rep. Prog. Phys. 67, 813 (2004)

28. M. Holler, F. Schapper, L. Gallmann, U. Keller, Phys. Rev. Lett. 106, 123601 (2011)

29. G. Sansone, F. Kelkensberg, J.F. Perez-Torres, F. Morales, M.F. Kling, W. Siu, O. Ghafur, P. Johnsson, M. Swoboda, E. Benedetti, F. Ferrari, Nature 465, 763 (2010)

30. F. Kelkensberg, W. Siu, J.F. Pérez-Torres, F. Morales, G. Gademann, A. Rouzée, P. Johnsson, M. Lucchini, F. Calegari, J.L. Sanz-Vicario, F. Martín, Phys. Rev. Lett. 107, 043002 (2011)

31. W. Siu, F. Kelkensberg, G. Gademann, A. Rouzée, P. Johnsson, D. Dowek, M. Lucchini, F. Calegari, U. De Giovannini, A. Rubio, R.R. Lucchese, Phys. Rev. A 84, 063412 (2011)

32. C. Neidel, J. Klei, C.H. Yang, A. Rouzée, M. Vrakking, K. Klünder, M. Miranda, C. Arnold, T. Fordell, A. L'Huillier, M. Gisselbrecht, Phys. Rev. Lett. 111, $033001(2013)$

33. F. Lépine, M.Y. Ivanov, M.J.J. Vrakking, Nat. Photonics 8, 195 (2014)

34. P. Rivière, O. Uhden, U. Saalmann, J.M. Rost, New J. Phys. 11, 053011 (2009)

35. C. Gao, P.M. Dinh, P.G. Reinhard, E. Suraud, Phys. Chem. Chem. Phys. 19, 19784 (2017)

36. D. Kiesewetter, R.R. Jones, A. Camper, S.B. Schoun, P. Agostini, L.F. DiMauro, Nat. Phys. 14, 68 (2017)

37. E. Runge, E.K. Gross, Phys. Rev. Lett. 52, 997 (1984)

38. M.A. Marques, C.A. Ullrich, F. Nogueira, A. Rubio, K. Burke, E.K. Gross, Lect. Notes in Phys. (Springer, Berlin, 2006)

39. C. Ullrich, Time-Dependent Density-Functional Theory: Concepts and Applications (Oxford University Press, 2012)

40. T. Brabec, P.M. Dinh, C. Gao, C. McDonald, P.G. Reinhard, É. Suraud, Eur. Phys. J. D 73, 212 (2019)

41. J.P. Perdew, Y. Wang, Phys. Rev. B 45, 13244 (1992)

42. J.P. Perdew, A. Zunger, Phys. Rev. B 23, 5048 (1981)

43. S. Yelin, E. Arimondo, C.C. Lin (serial editors), The twoset and average-density self-interaction corrections applied to small electronic systems, in Advances in Atomic, Molecular, and Optical Physics (2017), Vol. 64

44. C. Legrand, E. Suraud, P.G. Reinhard, J. Phys. B: At. Mol. Opt. Phys. 35, 1115 (2002)

45. P. Klüpfel, P.M. Dinh, P.G. Reinhard, E. Suraud, Phys. Rev. A 88, 052501 (2013)

46. S. Goedecker, M. Teter, J. Hutter, Phys. Rev. B 54, 1703 (1996)

47. S. Kümmel, M. Brack, P.G. Reinhard, Phys. Rev. B 62, 7602 (2000)

48. P.G. Reinhard, R. Cusson, Nucl. Phys. A 378, 418 (1982)

49. M. Feit, J. Fleck, A. Steiger, J. Comput. Phys. 47, 412 (1982)

50. P.G. Reinhard, P.D. Stevenson, D. Almehed, J.A. Maruhn, M.R. Strayer, Phys. Rev. E 73, 036709 (2006)

51. B. Montag, P.G. Reinhard, Z. Phys. D: At. Mol. Clusters 33, 265 (1995)

52. F. Calvayrac, P.G. Reinhard, E. Suraud, C.A. Ullrich, Phys. Rep. 337, 493 (2000)

53. P. Wopperer, B. Faber, P.M. Dinh, P.G. Reinhard, E. Suraud, Phys. Lett. A 375, 39 (2010) 
54. P. Wopperer, P.M. Dinh, E. Suraud, P.G. Reinhard, Phys. Rev. A 85, 015402 (2012)

55. C. Gao, P. Dinh, P. Reinhard, E. Suraud, On time-resolved electron emission at the attosecond time scale, in Journal of Physics: Conference Series (IOP Publishing, 2015), Vol. 635, p. 012007

56. F. Calvayrac, P.G. Reinhard, E. Suraud, Ann. Phys.
(N.Y.) 255, 125 (1997)

57. U. Kreibig, M. Vollmer, in Optical Properties of Metal Clusters (Springer Science \& Business Media, 2013), Vol. 25

58. C.Z. Gao, P.M. Dinh, P.G. Reinhard, E. Suraud, Ann. Phys. 360, 98 (2015)

59. P.G. Reinhard, L. Guo, J.A. Maruhn, Eur. Phys. J. A 32, 19 (2007) 